\title{
Tuning electronic reconstructions at the cuprate-manganite interface
}

\author{
C. Schlueter ${ }^{1,2,{ }^{*}}$ C. Aruta, ${ }^{3}$ N. Yang, ${ }^{4}$ A. Tebano, ${ }^{3,5}$ D. Di Castro, ${ }^{3,5}$ G. Balestrino, ${ }^{3,5}$ and T. L. Lee \\ ${ }^{1}$ Diamond Light Source Ltd., Harwell Science and Innovation Campus, Didcot, Oxfordshire, United Kingdom \\ ${ }^{2}$ Photon Science, Deutsches Elektronen-Synchrotron DESY, Notkestraße 85, 22607 Hamburg, Germany \\ ${ }^{3}$ CNR-SPIN, c/o Università di Roma Tor Vergata, Via del Politecnico 1, I-00133 Roma, Italy \\ ${ }^{4}$ School of Physical Science and Technology, ShanghaiTech University, 393 Middle Huaxia Road, Pudong, 201210 Shanghai, China \\ ${ }^{5}$ Dipartimento di Ingegneria Civile e Ingegneria Informatica, Università di Roma Tor Vergata Via del Politecnico 1, I-00133, Roma, Italy
}

(Received 13 February 2019; revised manuscript received 21 June 2019; published xxxxxx)

\begin{abstract}
The electronic properties of $\mathrm{CaCuO}_{2} / \mathrm{La}_{0.7} \mathrm{Sr}_{0.3} \mathrm{MnO}_{3}$ (LSMO) superlattices are determined by the electronic structure of the structural units and in particular their interfaces. The electronic structure of LSMO is governed by a metal-insulator transition, which is controlled by the thickness of the units and the sample temperature, resulting in a systematic downward band shift for metallic samples (i.e., thick LSMO units, low temperature). We present a systematic study of the changes in the valence-band structure and screening features in Mn $2 p$ and $\mathrm{Cu} 2 p$ core-level spectra. The results show that hybridization of $\mathrm{Cu} 3 d$ orbitals with out-of-plane $\mathrm{O} 2 p$ orbitals can be systematically tuned by controlling the band alignment at the interface via the metal-to-insulator transition of the LSMO units. This opens a new route to rational design of functional interfaces and control of orbital reconstructions.
\end{abstract}

DOI: 10.1103/PhysRevMaterials.00.004400

\section{INTRODUCTION}

A large variety of phases with a wide spectrum of properties are observed in transition-metal oxides. Charge transfer and magnetic interactions between metal ions are sensitive to the occupation of $d$ orbitals and their interaction with the ligand atoms [1]. Therefore, changes in the magnetic order, electron density, ligand position, and chemical bonding at the interfaces (IFs) of oxide heterostructures can result in electronic reconstruction phenomena. Particularly oxygen plays a key role in determining surface [2,3] and interface [4-6] electronic structure in transition-metal oxide heterostructures. The observation of high- $\mathrm{T}_{C}$ superconductivity $[7,8]$ at the IF of insulating cuprates, the magnetic coupling between cuprates and manganites [9], and oxygen redistribution at the IF between infinite-layer cuprates and strontium titanate [10] are but a few examples of such IF phenomena involving cuprates. For a rational design of heterostructures with engineered physical properties the control of orbital reconstructions at IFs is of crucial importance.

An interesting model system for the study of orbital reconstructions at oxide IFs are superlattices (SLs) comprised of the infinite-layer cuprate $\mathrm{CaCuO}_{2}(\mathrm{CCO})$ and optimally doped $\mathrm{La}_{0.7} \mathrm{Sr}_{0.3} \mathrm{MnO}_{3}$ (LSMO) [11]. In CCO, layers of $\mathrm{Cu}$ in fourfold planar oxygen coordination are separated by bare $\mathrm{Ca}$ atoms. This structural simplicity makes data interpretation less ambiguous compared to other infinite-layer cuprates [12-14]. LSMO has attracted a notable interest over the past decades due to phenomena such as colossal magnetoresistance $[15,16]$, tunneling magnetoresistance $[17,18]$, half metallicity [19], and a metal-insulator transition (MIT) above

\footnotetext{
*Corresponding author: christoph.schlueter@ desy.de
}

room temperature (RT). In thin films preferential $3 d e_{g}\left(z^{2}-\right.$ $r^{2}$ ) occupation at the IF to the substrate may lead to a suppression of the double exchange mechanism and the formation of a "dead-layer" region with suppressed conductivity [20]. Consequently, in mixed manganite multilayers a rich variety of electronic phases can be stabilized as a function of the thickness of individual blocks and the temperature [21]. Nevertheless, the origin of the dead-layer formation in LSMO thin films is still under debate [22]. Possible mechanisms likely playing a role are substrate-induced strain and structural distortions [23,24], chemical impurities and vacancies [25], as well as intermixing at the IF [26].

In the case of a CCO/LSMO superlattice two alternating atomic stacking sequences occur at the IFs [see Figs. 1(a) and 1(b)]: a $\mathrm{CuO}_{2}-(\mathrm{La}, \mathrm{Sr}) \mathrm{O}$ IF (type A) and a $\mathrm{Ca}-\mathrm{MnO}_{2}$ IF (type $\mathrm{B})$. The latter has the possibility to accommodate additional oxygen in the IF Ca plane, leading to $\mathrm{CaO}_{x}$ with $0<x<1$ as indicated in Fig. 1(b).

In analogy to the purely ionic mechanism proposed at the IF $\mathrm{TiO}_{2}-\mathrm{SrO}-\mathrm{AlO}_{2}-\mathrm{LaO}$ in the $\mathrm{LaAlO}_{3} / \mathrm{SrTiO}_{3}$ (STO) system [27], also for the CCO/LSMO structure a diverging built-in electrostatic potential is expected. Being confined in the $\mathrm{Mn}$ sublattice the mobile charges can only partially screen the diverging potential [28]. Therefore, screening by cation intermixing at the IF may occur. However, an oxygen redistribution process in the alkaline-earth metal IF planes is more likely since this process was reported for the structurally similar case of CCO/STO SLs [29]. Growth in strongly oxidizing conditions (SOCs) further favors incorporation of oxygen ions in the $\mathrm{Ca}$ plane of type-B IFs [8]. This additional oxygen would be apical for $\mathrm{Mn}$ and $\mathrm{Cu}$ at both sides of the IF [see Fig. 1(b)].

The amount of hybridized oxygen sites at the IF can strongly alter the overall electronic properties of an interface. 
(a)

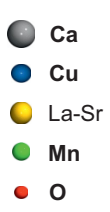

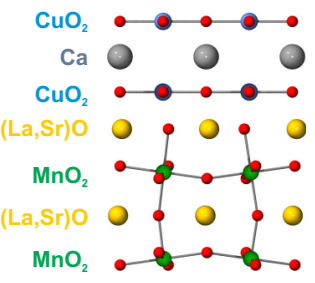

(b)

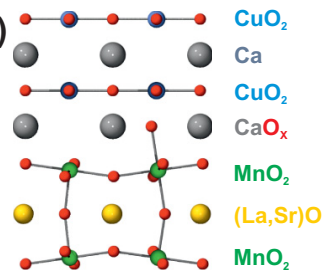

FIG. 1. The structure at $\mathrm{CaCuO}_{2} / \mathrm{La}_{0.7} \mathrm{Sr}_{0.3} \mathrm{MnO}_{3}$ interfaces is defined by the matching oxygen sublattices. Depending on the termination of the individual blocks there are two possible configurations: (a) a $\mathrm{CuO}_{2}-(\mathrm{La}, \mathrm{Sr}) \mathrm{O}$ interface (type $\mathrm{A}$ ) and (b) a Ca- $\mathrm{MnO}_{2}$ interface (type $\mathrm{B}$ ). In the second case, additional oxygen can be incorporated in the $\mathrm{Ca}$ planes. This leads to a $\mathrm{Ca}_{x}-\mathrm{MnO}_{2}$ IF with additional bridging oxygen between the two structural units.

In CCO/STO heterostructures the oxygen pressure during the growth process influences the amount of oxygen incorporated at the IFs $[8,13,14,29]$. In the $\mathrm{CCO} / \mathrm{STO}$ system, SLs prepared under SOC are superconducting with a $\mathrm{T}_{C}$ of up to $50 \mathrm{~K}$ [14]. In the CCO/LSMO system, SLs prepared under SOCs show a suppression of the MIT [11]. The exact role of oxygen uptake is still not completely understood. X-ray absorption spectroscopy (XAS) and x-ray linear dichroism (XLD) measurements indicate charge localization at IF and hybridization of $\mathrm{Cu} 3 d_{z^{2}-r^{2}}$ and $\mathrm{Mn} 3 d_{z^{2}-r^{2}}$ orbitals via IF O $2 p$ states. Furthermore, despite the dramatic changes in the transport properties of LSMO at its MIT, a systematic study for these SLs with different LSMO thickness and sample temperature is lacking. Here we attempt to fill this gap by using photoelectron spectroscopy to monitor the electronic structure of both the cuprate and the manganite units for a series of LSMO thicknesses with strong variations in their electronic properties.

Conventional photoelectron spectroscopy of buried IFs in oxide SLs is hindered by the small escape depth of the photoelectrons. It is well known that photoelectrons excited by photons in the hard $\mathrm{x}$-ray range can give access to the electronic structure of buried IFs $[4,30]$. The combination of valence-state contributions from all structural units renders the direct interpretation of the valence-band (VB) features ambiguous, particularly for a multielement system. On the other hand, $2 p$ spectra of transition-metal compounds (e.g., $\mathrm{Ni}, \mathrm{Mn}, \mathrm{Cu}$ ) [31] display screening features activated in response to the core hole formation in the photoemission process. These screening channels are highly sensitive to the valence electronic structure in their surroundings and thereby offer an elegant probe in case a direct VB observation is not feasible. It was previously shown that $\mathrm{Cu} 2 p$ spectra reflect the dimensionality in the $\mathrm{CuO}_{4}$ plaquettes [32] and react most sensitively to metal-ligand charge transfer and hybridization [33]. Recently it was shown how Mn $2 p$ spectra reflect hybridization strength, metallicity, and magnetic properties [22]. Hard x-ray photoelectron spectroscopy (HAXPES) is therefore the technique of choice for the study of the electronic structure of buried IFs.

In this paper we study a set of CCO/LSMO SLs with CCO thickness fixed at three monolayers (MLs). The LSMO thickness is varied systematically through the range where the MIT is expected for thin films. We provide evidence of

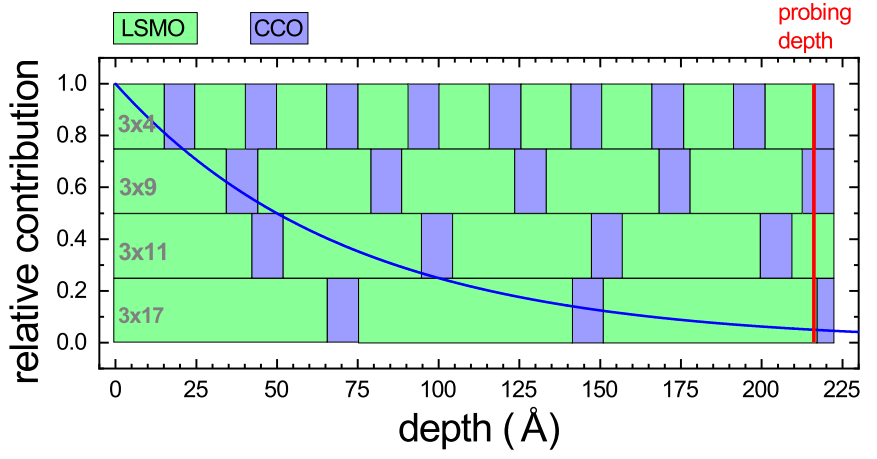

FIG. 2. The relative intensity contribution to the total signal measured by HAXPES (blue line) as a function of the depth in the sample. The red line shows the expected probing depth. The boxes show schematic sketches of the four different $\mathrm{CCO}_{n} / \mathrm{LSMO}_{m}$ SLs: $n \times m=3 \times 4,3 \times 9,3 \times 11$, and $3 \times 17$.

extra apical oxygen entering the IFs resulting in an increased coordination number of the interfacial $\mathrm{Cu}$ atoms. Moreover, the electronic properties of these SLs can be tuned through the MIT by varying the temperature as well as the LSMO thickness. We demonstrate the influence of modified electronic structure in the LSMO units on screening effects observed in $\mathrm{Cu} 2 p$ and Mn $2 p$ HAXPES core-level spectra and provide complementary data by XAS and XLD. Our data reveal a systematic variation of the band alignment and the $\mathrm{Cu} 3 d-\mathrm{O}$ $2 p$ orbital hybridization at the IF.

\section{EXPERIMENTAL DETAILS}

$\mathrm{CCO} / \mathrm{LSMO}$ SLs were grown on A-site-terminated $\mathrm{NdGaO}_{3}$ (110) (NGO) substrates by pulsed laser deposition. Here we study a sequence of $\mathrm{CCO}_{n} / \mathrm{LSMO}_{m}$ SLs with $n=$ 3 and $m=4,9,11$, and 17 . The respective numbers of $\mathrm{CCO} / \mathrm{STO}$ bilayers, $N$, was chosen to be $N=20,16,15$, and 12 to obtain a comparable SL thickness well above the HAXPES probing depth. The buildup of the SLs is depicted schematically in Fig. 2. SLs were grown in SOCs using 1 mbar of oxygen containing $12 \%$ ozone and subsequently quenched to RT in 1 bar of oxygen. The detailed growth procedure was described previously [11]. One $\mathrm{n} \times \mathrm{m}=3 \times 4$ SL was prepared in moderately oxidizing conditions (MOCs) with an oxygen pressure lower than $0.1 \mathrm{mbar}$, which is the oxygen partial pressure normally used to grow optimally doped manganites [34]. This SL will be referred to as $3 \times 4 *$.

X-ray reflectivity, XAS/XLD, and HAXPES experiments were performed at beam line I09 of Diamond Light Source, Ltd. (Didcot, United Kingdom), where both soft- (110$2000 \mathrm{eV})$ and hard $(2.1-18 \mathrm{keV}) \mathrm{x}$-rays, delivered by APPLE II (HU60) and in-vacuum (U27) undulators, respectively, are both available at the experimental end station with a base pressure below $3 \times 10^{-10} \mathrm{mbar}$.

The $\mathrm{x}$-ray reflectivity was recorded in a $\pi$-scattering geometry by an in-vacuum photodiode at a photon energy of $2.5 \mathrm{keV}$. Cu $L_{3}$-edge XAS /XLD was performed at $60 \mathrm{~K}$ with linear horizontal and vertical polarized photons of the soft Xray branch. Samples were fixed at an incident angle of $15^{\circ}$. All scans were collected in a total electron yield mode and were 
normalized to the incident photon flux. HAXPES spectra were measured at a photon energy of $5.95 \mathrm{keV}$ using an EW4000 electron analyzer with an acceptance angle of $\pm 30^{\circ}$ (ScientaOmicron, Uppsala, Sweden) pointing in the polarization direction of the photon beam. We employed a Si(333) channel cut crystal to reduce the energy band pass of the photon beam. The overall experimental resolution is estimated to be $80 \mathrm{meV}$. The overall energy stability was better than $\pm 10 \mathrm{meV}$ in $24 \mathrm{~h}$. Photoelectron intensity was maximized by conducting HAXPES measurements at a grazing incident angle of $\sim 1^{\circ}$ leaving the sample in near normal emission geometry. The energy scale of each spectrum was calibrated against a $\mathrm{Au}$ Fermi level. Unless stated differently all HAXPES spectra are presented after subtraction of a Shirley-type background and subsequent normalization to their total intensity.

The significance of using HAXPES is illustrated in Fig. 2. The contribution $I_{0}$ to the total measured intensity $I$ by material below a depth $d$ is described by a Lambert-Beer equation $I=I_{0} \times e^{\frac{-d}{\lambda \sin \theta}}$. Here, $\theta$ is the emission angle, which in our case was $\sim 90^{\circ}$. The inealastic mean free path $\lambda$ is estimated for valence electrons excited by $5.95 \mathrm{keV}$ photons [35]. The blue line in Fig. 2 shows the relative intensity contribution as a function of depth. The red line marks the probing depth $(3 \lambda)$ where $95 \%$ of the total intensity originates. The probing depths are $250 \AA$ and $223 \AA$ for CCO and LSMO, respectively. For higher-binding-energy (lower-kinetic-energy) electrons such as those originating from a $\mathrm{Cu} 2 p$ core level, these values reduce only slightly to 218 and $193 \AA$ A, respectively. In Fig. 2 the blue curve is underlain by a schematic of the structures of the SLs to underline the necessity of employing HAXPES to access the buried cuprate blocks and to gather signal from buried interfaces.

\section{RESULTS}

\section{A. Structural characterization}

Figure 3(a) shows x-ray reflectivity measured for $2.5 \mathrm{keV}$ photons for the $3 \times 4$ SL with 20 repetitions. To model the reflectivity, the thickness and roughness of each layer were allowed to vary freely. Additionally, the electron density of the $\mathrm{CCO}$ layer was varied between $\mathrm{CaCuO}_{2}$ and a nominal $\mathrm{CaCuO}_{3}$ and an adsorbate layer was added to the surface of the SL. The mean values of the roughness and thickness obtained from the model are summarized in Table I. The real and imaginary parts of the scattering-length density (SLD) of the model are visualized in Fig. 3(b). Figure 3(c) shows the thickness of the individual blocks in units of MLs as obtained from the fit. It can be seen that the fit yields preferentially integer and half-integer numbers of MLs. The calculated IF roughness of $\sim 2-3 \AA$ clearly demonstrates the formation of sharp CCO/LSMO IFs and confirms our previous results obtained from scanning transmission electron microscopy (STEM) on similar samples [11]. The best agreement with the data was found for a CCO layer with increased density corresponding to a nominal stoichiometry of $\mathrm{CaCuO}_{2.65}$. This increase in electron density of the CCO layers is interpreted as first evidence in support of the concept of oxygen uptake at the CCO/LSMO IF.
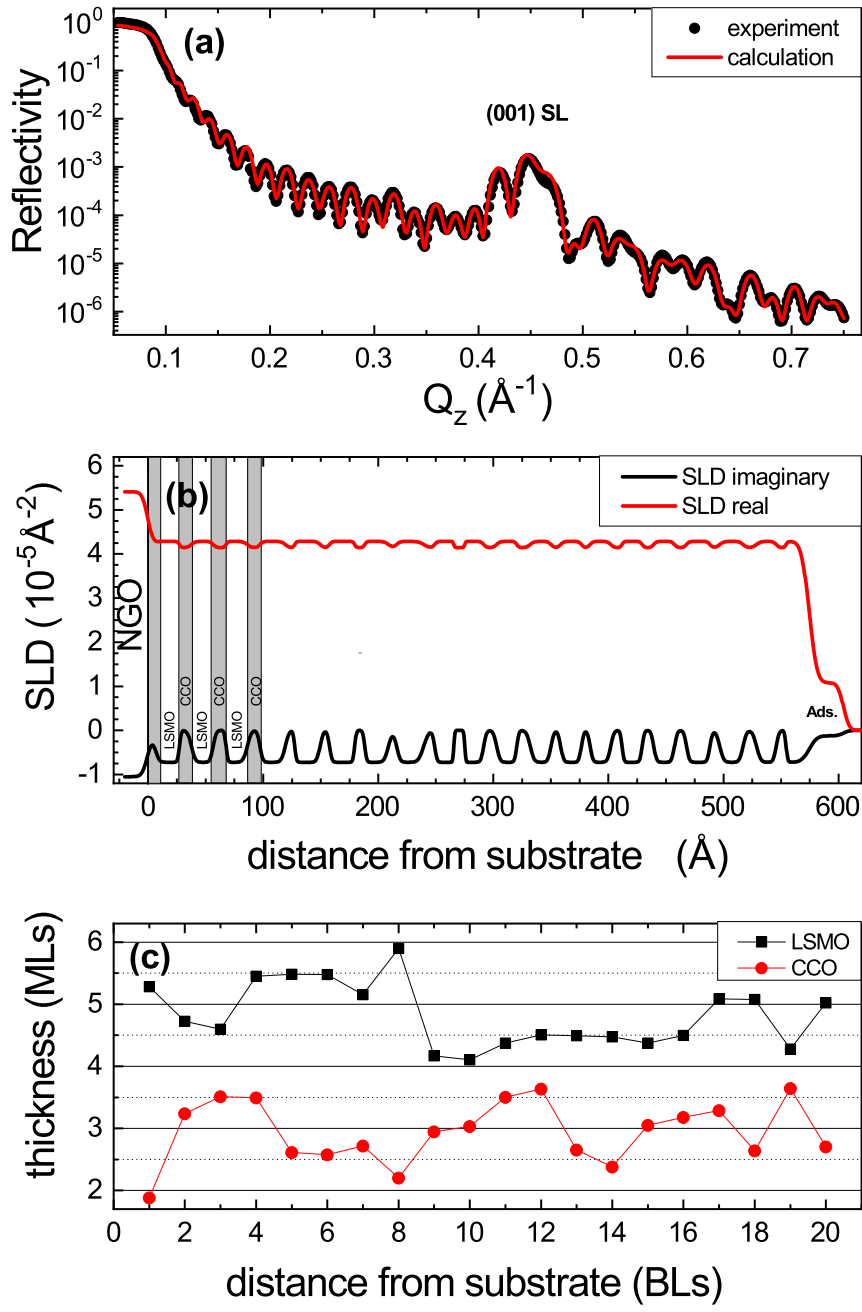

FIG. 3. Reflectivity of a $3 \times 4$ SL with $20 \mathrm{CCO} / \mathrm{LSMO}$ bilayer (BL) repetitions: (a) experimental data and calculated reflectivity, (b) real and imaginary part of the SLD for the model used in the calculation, and (c) thickness in MLs of the individual CCO and LSMO blocks as a function of distance from the substrate in $\mathrm{CCO} / \mathrm{LSMO}$ BLs.

\section{B. Uptake of apical oxygen}

Figure 4(a) shows the $\mathrm{Cu} L$-edge XAS spectra with the electric field vector $E$ of the incident photon beam parallel to the $c$ and $a / b$ axes of the SLs for the $3 \times 4$ and $3 \times 4 *$ SLs. The peak observed at $932.8 \mathrm{eV}$ corresponds to a transition from the $\mathrm{Cu} 2 p$ level to the hybridized $\mathrm{Cu} 3 d-\mathrm{O} 2 p$ antibonding state and is thus sensitive to the presence of oxygen ligands in the photon polarization direction [9]. For the SLs to remain

TABLE I. Summary of the average roughness and thickness obtained from the model refinement of the reflectivity measurements displayed in Fig. 3.

\begin{tabular}{lccc}
\hline \hline & $\sigma(\AA)$ & $d(\AA)$ & $d(\mathrm{ML})$ \\
\hline LSMO & $2.0 \pm 0.64$ & $19.3 \pm 2.06$ & $4.82 \pm 0.52$ \\
CCO & $2.9 \pm 0.5$ & $9.4 \pm 1.6$ & $2.94 \pm 0.54$ \\
\hline
\end{tabular}



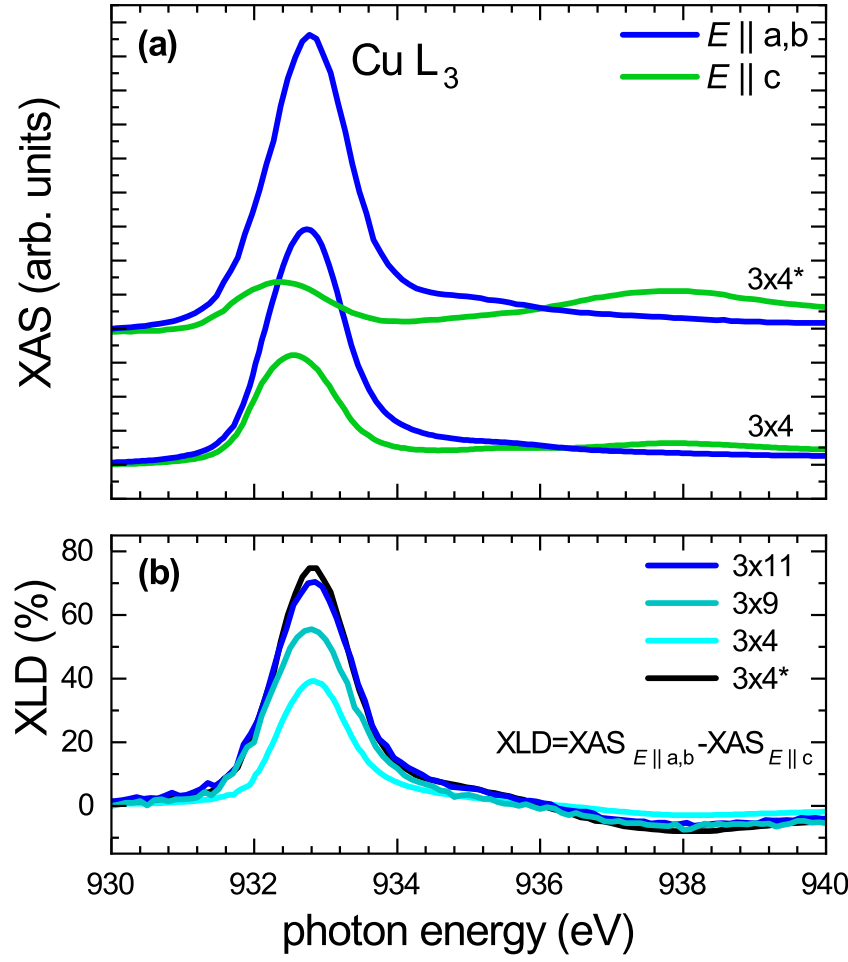

FIG. 4. (a) Normalized $\mathrm{Cu} L_{3}$-edge XAS spectra measured in a total electron yield mode at $60 \mathrm{~K}$ with $E \| c$ and $E \| a / b$ for the $3 \times 4$ and $3 \times 4 *$ SLs confirms uptake of oxygen for SOCs and (b) x-ray linear dichroism spectra measured at $60 \mathrm{~K}$ for SLs of various LSMO thicknesses.

stoichiometric, the top and bottom IFs of each CCO layer need to be of the different configurations depicted in Fig. 1. This leads to one single apical oxygen atom at the type-A IF per three $\mathrm{CCO}$ unit cells. From the coordination number of $\mathrm{Cu}$ the XAS peak at $932.8 \mathrm{eV}$ is thus expected to yield an intensity ratio of 1:6 between the measurements with $E \| c$ and $E \| a, b$. This ratio is indeed observed for the $3 \times 4 * \mathrm{SL}$, which was prepared in MOCs. For the SOCs $3 \times 4$ SL the intensity ratio increases to 0.45 , which is close to the value of 0.33 expected for an additional apical oxygen atom incorporated per unit cell at the type-B IF. Here we would like to point out that a ratio of 1 as observed for similar interfaces [9] can only be observed when all $\mathrm{Cu}$ atoms of the cuprate unit were fully octahedrally coordinated.

The XAS data therefore support a scenario in which additional oxygen is incorporated at the CCO/LSMO IFs which is apical to $\mathrm{Cu}$, for the SLs prepared under SOCs. This uptake of oxygen is also suggested by the x-ray reflectivity analysis in Fig. 3 on the $3 \times 4 \mathrm{SL}$, where the oxygen content in the $\mathrm{CCO}$ is found to be $33 \%$ higher than the bulk value, i.e., $\mathrm{CaCuO}_{2.65}$ instead of $\mathrm{CaCuO}_{2}$. The amount of bridging oxygen is expected to be constant for all SOC samples due to the identical growth conditions. First-principles total energy calculations of the IF structure of CCO on STO [36] show that, when the CCO is two unit cells or thinner, the polar electrostatic instability in the $\mathrm{CCO}$ layer may give rise to a chain-type structure, where the oxygen at the type-A IF moves from the $\mathrm{Cu}$ plane to the adjacent $\mathrm{Ca}$ plane. Such a

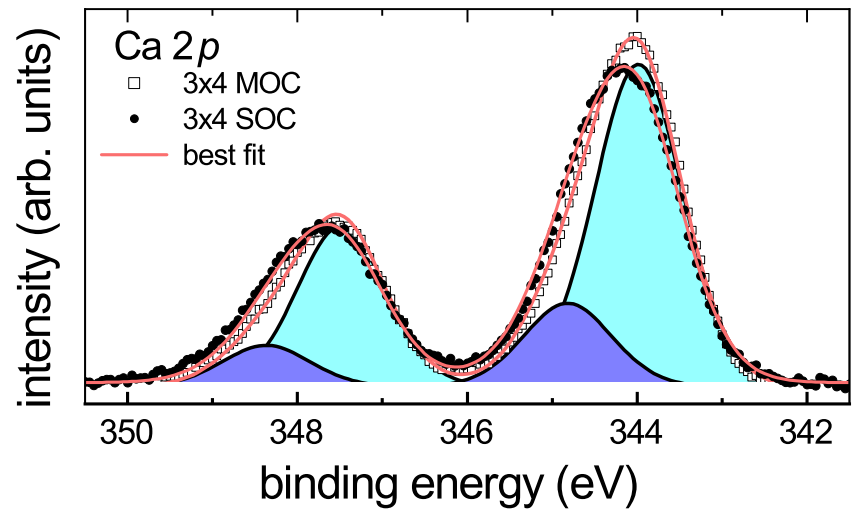

FIG. 5. Ca $2 p$ HAXPES spectra of the MOCs (open squares) and SOCs (solid circles) $3 \times 4$ SLs at $60 \mathrm{~K}$. The best fit with two spinorbit split doublets is shown with a red continuous line. The change in line shape is indicative of an oxygen incorporation at the interface.

reconstruction would transform the $\mathrm{Cu}$ at the IF from being fivefold oxygen coordinated to a fourfold coordination in a vertical plane, resulting in a high $\mathrm{Cu} L$-edge XAS intensity ratio between the out-of-plane $(E \| c)$ and in-plane $(E \| a, b)$ polarizations contrary to the data presented above.

In Fig. 4(b) the XLD observed for the $3 \times 4,3 \times 9$, and $3 \times 11$ SLs is shown together with that of the $3 \times 4 *$ SL. The XLD is the difference between the XAS in- and out-of-plane components, $\mathrm{XAS}_{E \| a, b}-\mathrm{XAS}_{E \| c}$ and gives information of the occupancy of the $\mathrm{Cu} 3 d$ orbitals. The observed XLD signal increases systematically with increasing LSMO thickness. This may be due to (i) a diffusion-limited oxygen uptake process or (ii) a change in the $\mathrm{Cu} 3 d-\mathrm{O} 2 p$ hybridization. Further discussion in favor of $\mathrm{Cu} 3 d-\mathrm{O} 2 p$ hybridization is given in conjunction with the $\mathrm{Cu} 2 p$ HAXPES spectra (see Fig. 11).

The oxidation of the CCO interfaces under SOCs is further supported by comparison of Ca $2 p$ HAXPES spectra shown in Fig. 5 for the $3 \times 4 *$ and $3 \times 4$ SLs. The spinorbit split $2 p_{3 / 2}$ and $2 p_{1 / 2}$ doublet has an asymmetric peak shape. Spectra were modeled with two doublets of a mixed Gaussian-Lorentzian line shape. Analysis shows an increase of the higher-binding-energy component, which corresponds to $\mathrm{Ca}$ in a higher oxidation state, as a more oxidizing growth condition is applied. Consistent with the formation of extra oxygen apical to the copper, the SOC is expected to give rise to Ca with a planar oxygen coordination at the type-B IF. In a previous study [29] we have also reported additional $\mathrm{Ca} 2 p$ components on the high-binding-energy side for $\mathrm{CCO} / \mathrm{STO}$ SLs prepared in SOCs.

The data presented so far give evidence of the uptake of additional oxygen in the SOC samples. Our recent publication [11] was able to show by $\mathrm{Mn} L$-edge absorption spectra an increase of out-of-plane covalency by bonding to $\mathrm{Cu}$ orbitals via IF oxygen. The intensity ratio of $\mathrm{Cu} L$-edge XAS parallel and perpendicular to the sample surface is supporting the idea that the additional oxygen is confined to the IF region. We note that the ratios perfectly agree with the ones expected for an overoxidized interface. The planar oxygen in the interfacial $\mathrm{Ca}$ plane is hence a bridging oxygen between $\mathrm{Cu}$ and $\mathrm{Mn}$. 


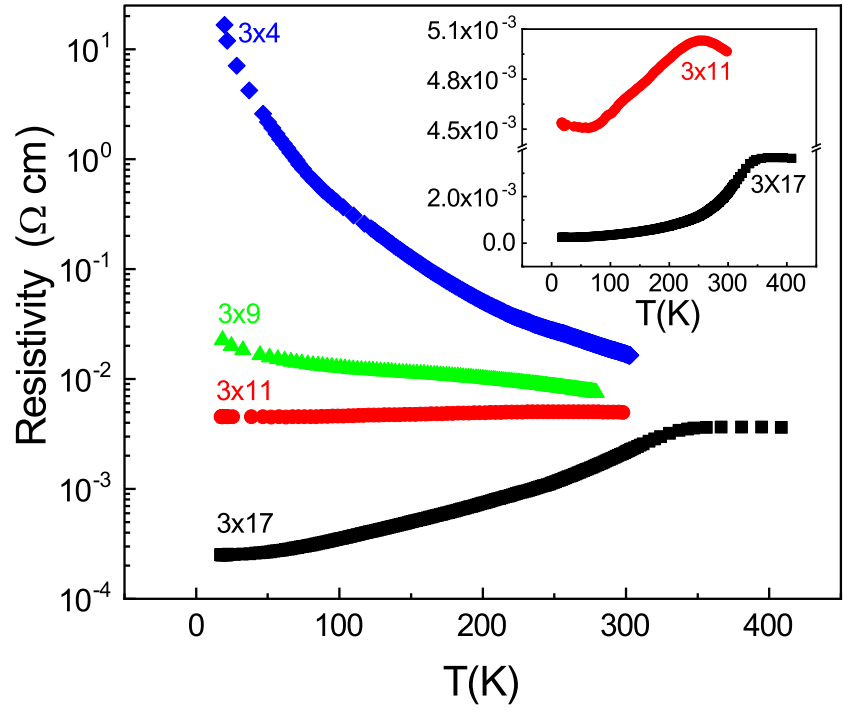

FIG. 6. Resistivity as a function of temperature in semilogarithmic scale for $\mathrm{CCO}_{3} / \mathrm{LSMO}_{m}$ SLs grown in SOC: $m=17$ (black squares), $m=11$ (red circles), $m=9$ (green triangles), and $m=4$ (blue diamonds). The linear scale plot of the data of the SLs with $m=17$ and $m=11$ is reported in the inset.

\section{Changes in the LSMO blocks as a function of thickness and temperature}

Resistivity versus temperature is reported in Fig. 6. The $3 \times 17$ SLs show a MIT above RT, where the system goes from a paramagnetic insulating phase to a ferromagnetic metallic phase. For the SLs with a thinner LSMO block of 11 MLs the MIT reduces rapidly to $255 \mathrm{~K}$, as can be observed in the inset of Fig. 6. As previously reported [25], below the MIT the transport mechanism is well described by a variable range hopping model, while above the MIT the transport mechanism is well described by a polaronic activated mechanism. In the $3 \times 11 \mathrm{SL}$ possible charge localization effects give rise to the minimum observed in the resistivity curve reported in the inset of Fig. 6 . When the LSMO is only nine MLs thick the SL goes from a paramagnetic to a semiconducting phase. The $3 \times 4$ SL shows a semiconducting behavior in the whole accessible temperature range. The transport properties of the SLs depend therefore strongly on the LSMO thickness. These transport measurements agree well with our previous reports [11] and data for LSMO thin films [34].

Further relevant information on the electronic structure is obtained from the VB photoelectron spectra. To identify the Mn 3d-related features we measured VB spectra at different excitation energies - off and on the Mn $2 p$ to $3 d$ resonance (not shown). We observe a strong enhancement of spectral weight close to the Fermi level, which could be identified as the Mn $3 d t_{2 g}$ and $e_{g}$ states based on the previous resonant photoemission studies on LSMO thin films [37]. The corresponding features are present in the VB HAXPES spectra measured with $5.95 \mathrm{keV}$ photon energy at $60 \mathrm{~K}$ shown in Fig. 7. The spectra appear to be dominated by the spectral features of LSMO. The maximum around $6 \mathrm{eV}$ corresponds to $\mathrm{O} 2 p$ and the signature of the $\mathrm{Mn} 3 d t_{2 g}$ and $e_{g}$ states is clearly visible between 0 and $3 \mathrm{eV}$. Comparison of the

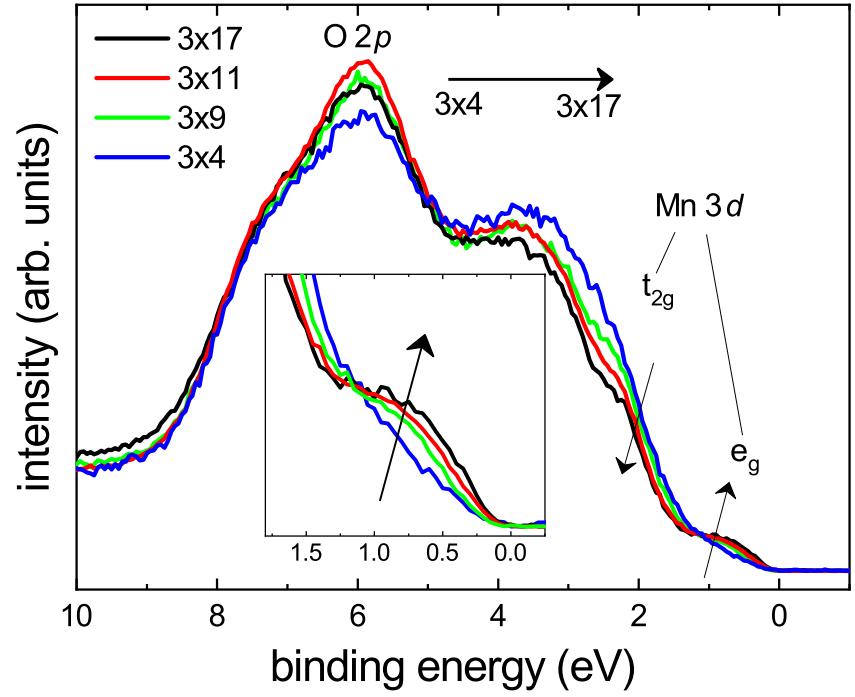

FIG. 7. HAXPES spectra of the valence-band region for a sequence of CCO/LSMO SLs at $60 \mathrm{~K}$. The samples are $3 \times 17$ (black), $3 \times 11$ (red), $3 \times 9$ (green), and $3 \times 4$ (blue). The inset shows in detail the spectral region 1.6 to $-0.2 \mathrm{eV}$ binding energy where an increase in spectral weight is visible close to the Fermi level. The arrows indicate the increase in LSMO layer thickness. For better comparability spectra are normalized to the total intensity.

different SLs shows a relative shift of spectral weight from $t_{2 g}$ states to the $e_{g}$ states for SLs with thicker LSMO blocks, i.e., for more-conducting samples.

Figure 8(a) shows Mn $2 p$ HAXPES spectra recorded at $60 \mathrm{~K}$. The main peak at $641 \mathrm{eV}$ exhibits a shoulder at the low-binding-energy side which is weaker for SLs with thinner LSMO blocks and disappears completely for the $3 \times 4 \mathrm{SL}$. Figure $8(\mathrm{~b})$ shows the temperature dependence for the two extreme SLs $(3 \times 4$ and $3 \times 17)$. No influence of temperature is observed for the $3 \times 4 \mathrm{SL}$, while for the $3 \times 17 \mathrm{SL}$ the

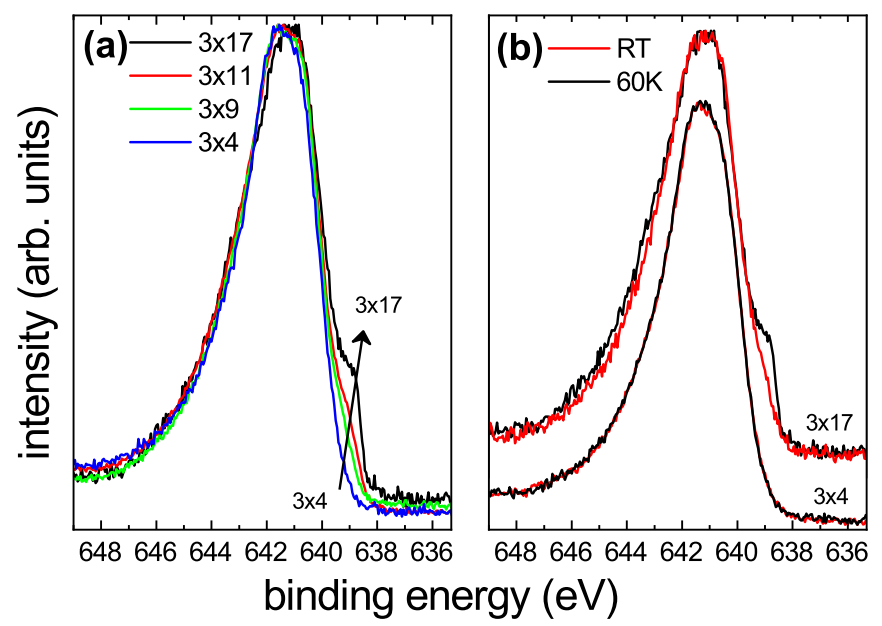

FIG. 8. Mn $2 p$ spectra for (a) a sequence of different $n / m$ ratios where the thickness of the LSMO block is increased step by step at $60 \mathrm{~K}$ and (b) a comparison for measurements at RT and $60 \mathrm{~K}$ for the two extreme SLs, $3 \times 4$ and $3 \times 17$. The "well-screened" satellite can be clearly identified in the metallic SLs. 


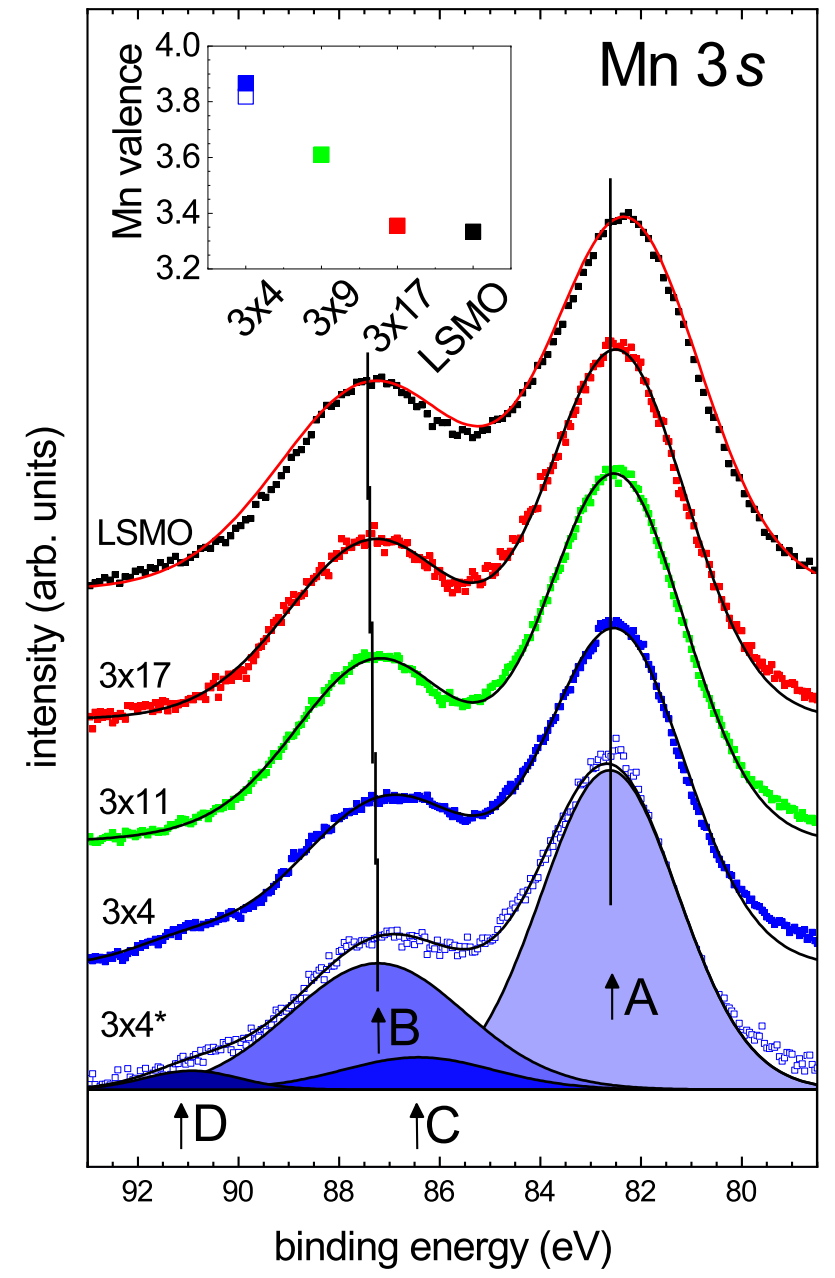

FIG. 9. Mn $3 s$ HAXPES spectra measured at $60 \mathrm{~K}$ for different LSMO thickness in comparison with an optimally doped LSMO reference sample. The individual components are shown on the bottom of the figure for the $3 \times 4 *$ SL. The inset depicts the Mn mixed-valence state associated with the exchange splitting.

shoulder is significantly weaker in RT measurements. It is well known for LSMO thin films that Mn $2 p$ core levels show a "well-screened" state at their lower-binding-energy side in HAXPES spectra. This feature is understood as a result of screening by hybridized states between the transitionmetal $3 d$ states and a coherent metallic band close to $E_{F}$ [38]. It was shown experimentally that the strength of this screening feature correlates with doping level and hence transport properties of LSMO thin films [25,38]. The suppression of the well-screened state indicates a weaker $\mathrm{Mn} 3 d-\mathrm{O} 2 p$ hybridization in the less-conducting samples with a thinner LSMO unit.

Figure 9 represents Mn 3s HAXPES spectra measured at $60 \mathrm{~K}$ for the various SLs. The two main peaks A and $\mathrm{B}$ are associated with the high- and low-spin final states, respectively, resulting from the well-established exchange interaction between the $3 s$ core hole and the $3 d$ electrons. The magnitude of the $3 s$ exchange splitting reflects the local magnetic moment and hence the valence states of the Mn [39]. Using the linear dependence of the valence on the $3 s$ splitting
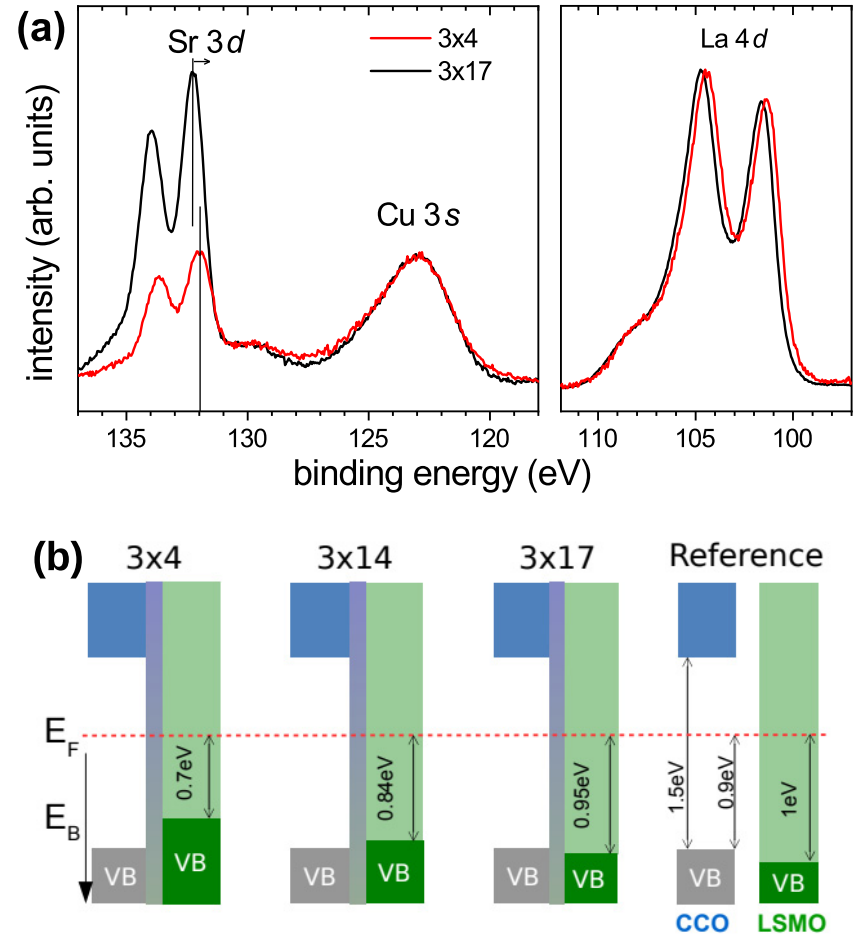

FIG. 10. (a) HAXPES core-level spectra of $\mathrm{Sr} 3 d, \mathrm{Cu} 3 s$, and La $4 d$ at $60 \mathrm{~K}$ for selected SLs. LSMO core-level spectra show a shift as a function of LSMO thickness. The energy scale of each spectrum was calibrated against a Au Fermi level. (b) The band alignment estimated from the core-level spectra depicted above as a function of LSMO layer thickness.

reported in Ref. [40] we estimate the Mn valence gradually decreases for SLs with thicker LSMO blocks from 3.86 for the $3 \times 4$ SL to 3.36 in an optimally doped, thick LSMO film. We also observed additional components $\mathrm{C}$ and $\mathrm{D}$, on the high-binding-energy side which are most pronounced in the SLs with thinner LSMO blocks. Similar features have been reported in the literature for other manganese compounds $[41,42]$. To identify the exact origin of these weak features for the SLs will require further study and is beyond the scope of this publication.

Finally, in Fig. 10 (a) we show the core-level HAXPES spectra of $\mathrm{Sr} 3 d, \mathrm{Cu} 3 s$, and $\mathrm{La} 4 d$ for the $3 \times 4$ and $3 \times 17$ SLs at $60 \mathrm{~K}$. An energy shift of $\sim 250 \mathrm{meV}$ of the LSMO core levels with the LSMO thickness is clearly visible, while no corresponding shift is detected for the CCO core levels. From the relative peak shifts we estimate the band alignment between the CCO and LSMO blocks as shown schematically in Fig. 10(b) by following the procedure described in the literature for other oxides $[43,44]$. Here the energy shift of the LSMO bands is represented by a change in the energy separation between the $E_{F}$ and the leading edge of the LSMO $t_{2 g}$ band. Figure 10(b) thus shows a clear downwards shift of the LSMO bands relative to the CCO bands in the SLs with thicker LSMO units, suggesting that electronic states close to the Fermi level are gradually filled by more localized electrons towards thicker LSMO blocks. 


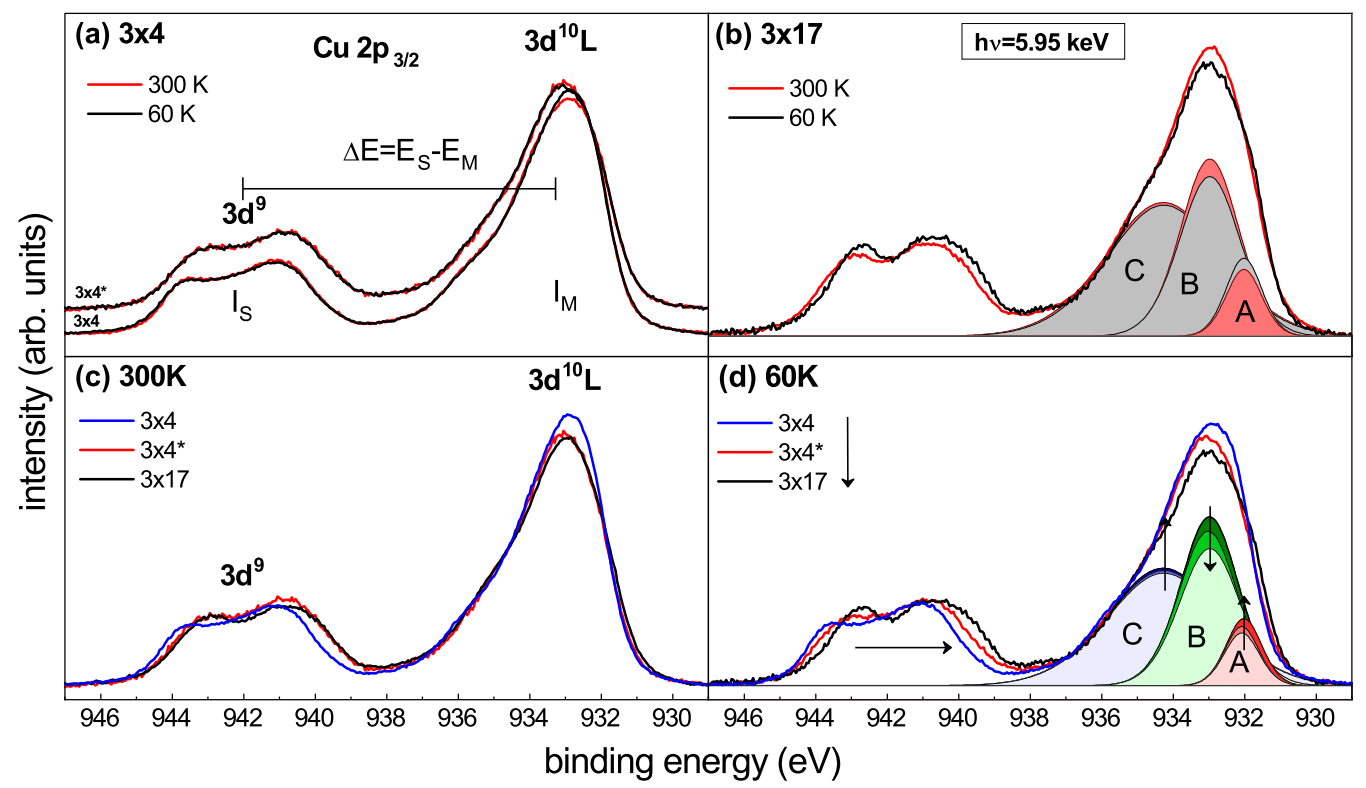

FIG. 11. Comparison of $\mathrm{Cu} 2 p$ spectra measured at $5.95 \mathrm{keV}$ for (a) the $3 \times 4$ and $3 \times 4 *$ SLs and (b) the $3 \times 17$ SL at RT (red lines) and $60 \mathrm{~K}$ (black lines). Comparison of (c) the $\mathrm{Cu} 2 p$ spectra at RT and (d) the spectra at $60 \mathrm{~K}$ for the $3 \times 4$ (blue line), the $3 \times 4 *$ (red line), and the $3 \times 17$ (black line) SLs. $\Delta E$ indicates the energy separation and $I_{S} / I_{M}$ is the intensity ratio between main peak and satellite. Additionally, the screening components $\mathrm{A}, \mathrm{B}$, and $\mathrm{C}$ are shown. The arrows indicate the change going from the $3 \times 4 \mathrm{SOC}$ to the $3 \times 4 * \mathrm{MOC}$, and $3 \times 17$ SLs.

\section{Changes in the CCO blocks: $\mathrm{Cu} 2 p$ screening and hybridization}

In Fig. 11 we compare the $\mathrm{Cu} 2 p$ HAXPES spectra for the $3 \times 4 *, 3 \times 4$, and $3 \times 17$ SLs at RT and $60 \mathrm{~K}$. The remaining SLs exhibit intermediate spectral features and are omitted from the figure for clarity. The four figure panels are organized to manifest the temperature dependence in Figs. 11(a) and 11(b) for the two LSMO thicknesses and the LSMO thickness dependence in Figs. 11(c) and 11(d) at RT and $60 \mathrm{~K}$.

The $\mathrm{Cu} 2 p_{3 / 2}$ component in Fig. 11 shows a strong asymmetric main peak at $933 \mathrm{eV}$ and a satellite about $8 \mathrm{eV}$ higher in binding energy. These features have been well studied experimentally and theoretically in the framework of superconducting cuprates $[32,45,46]$. The main peak is identified as a $2 p^{5} 3 d^{10} \mathrm{~L}$ final state, where $\mathrm{L}$ refers to a ligand hole, while the satellite is associated with a poorly screened $2 p^{5} 3 d^{9}$ final state; i.e., the hole occupies the $\mathrm{Cu} 3 d$ orbital.

The $\mathrm{Cu} 2 p_{3 / 2}$ spectra of the $3 \times 4$ and $3 \times 4 *$ SLs show significant differences [Fig. 11(a)]. For the $3 \times 4 *$ SL the energy position of the $3 d^{9}$ satellite shifts towards lower binding energy. The $3 d^{10} \mathrm{~L}$ main peak shifts towards higher binding energy and has a different line shape. These spectral differences are attributed to different oxidation conditions (MOCs vs SOCs), which is expected to modify the oxygen coordination at the IF. However, spectra measured at $60 \mathrm{~K}$ and RT show no detectable differences. By contrast, clear changes between RT and $60 \mathrm{~K}$ are observed for SLs that show clear spectral changes in the LSMO units such as the $3 \times 17 \mathrm{SL}$ in Fig. 11(b). There is a noticeable binding energy shift of the satellite accompanied by a shift of the spectral weight. Similar changes to the line shape, e.g., a shift of the $3 d^{9}$ satellite to lower binding energies, are also observed as functions of
LSMO thickness in Figs. 11(c) and 11(d) with the energy shift of the satellite being more pronounced at $60 \mathrm{~K}$.

Previously, the fine structure of $2 p$ core-level spectra was investigated theoretically by Bocquet and Fujimori [33] for divalent and trivalent $3 d$ transition-metal compounds. Their comprehensive study shows that the energy separation $\Delta E$ between the main peak and the satellite, and the intensity ratio $I_{S} / I_{M}$ of the two components, are a direct measure of the ligand- $2 p$ to metal- $3 d$ hybridization (or hopping energy) $T$, the ligand-to-metal charge transfer energy $\Delta$, and the interaction between the localized $3 d$ electrons, $U$. At stronger hybridization $T$, for example, satellites were found to be weaker (smaller $I_{S} / I_{M}$ ) and shifted in binding energy (larger $\Delta E$ ).

For a quantitative analysis of the $\mathrm{Cu} 2 p$ line shape as a function of temperature and LSMO thickness, we estimate the intensities $I_{S}$ and $I_{M}$ from the integral of the satellite and the main peak and $\Delta E$ from the centers of mass of the two components. In Fig. 12 we plot thus obtained $I_{S} / I_{M}$ versus $\Delta E$ values for all the SLs at RT and $60 \mathrm{~K}$ together with the values predicted by theory for $\mathrm{Cu}^{2+}$ with $U=6$ and $7 \mathrm{eV}$ and $\Delta=1 \mathrm{eV}$ [33]. Data points in the $I_{S} / I_{M}-\Delta E$ plot scatter along a sloped line parallel to the constant $\Delta$ and $U$ direction. This suggests that the evolution of the $\mathrm{Cu} 2 p$ spectrum is associated mainly with the variation of the $\mathrm{Cu}$ ligand hybridization strength $T$. As expected for the $3 \times 4 *$ SL grown in MOCs, the hybridization strength $T$ is weaker by $\sim 0.2 \mathrm{eV}$ with respect to the $3 \times 4 \mathrm{SL}$. The remaining SLs show a systematic dependence of $T$ on the temperature (a reduction of $0.15-0.2 \mathrm{eV}$ from RT to $60 \mathrm{~K}$ ) and LSMO thickness, both indicating a stronger hybridization for the less metallic samples. 


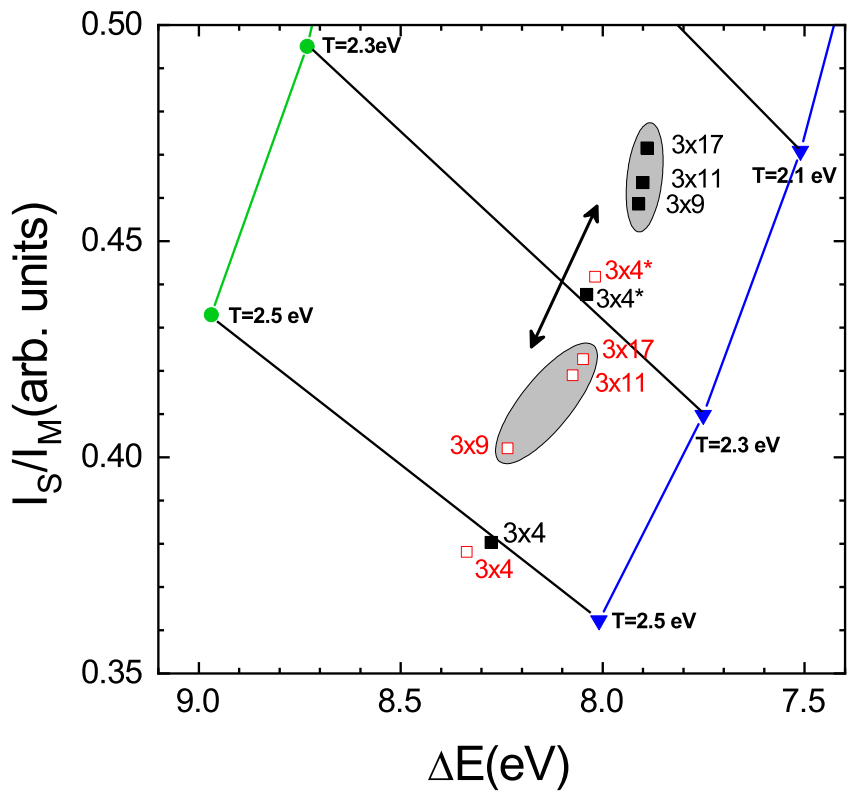

FIG. 12. $I_{S} / I_{M}$ ratio plotted versus $\Delta E$ shows a linear relationship. Solid black squares show the results for spectra measured at $60 \mathrm{~K}$, open red squares for RT spectra. Additionally we show the theoretical values taken from Bocquet and Fujimori [33]. Closed circles correspond to $U=7 \mathrm{eV}$ and $\Delta=1 \mathrm{eV}$, blue triangles to $U=6 \mathrm{eV}$ and $\Delta=1 \mathrm{eV}$. The values for $\mathrm{Cu} 3 d-\mathrm{O} 2 p$ hybridization strength $T$ are given next to the symbols.

The $2 p^{5} 3 d^{10} \mathrm{~L}$ main peak is composed of several components. In agreement with theoretical work $[45,47]$ we employed a multipeak deconvolution procedure, using three components (A, B, and C) with Gaussian-Lorentzian line shape to extract the peak positions and intensities. The peak positions and widths were obtained by fitting the sum of all measured spectra and were used to constrain the fits of the individual spectra within the boundaries of the experimental resolution.

After the photoemission process, the core hole can be located in the $2 p$ orbitals directly surrounding the $\mathrm{Cu}$ site (local screening, B and C) or on more distant $2 p$ orbitals surrounding a neighboring $\mathrm{Cu}$ atom (nonlocal screening, $\mathrm{A}$ ) $[46,48]$. The feature A increases with thickness of the LSMO block at the expense of components $\mathrm{B}$. The changes in $\mathrm{C}$ are only minor. The gradual increase of the nonlocal screening channel A is interpreted as a signature of the decreased charge localization at the IF as observed in the XAS/XLD spectra. The stronger nonlocal contribution reflects the decrease of electrons occupying the hybridized $3 d_{z^{2}-r^{2}}$ orbitals in favor of occupation of more localized in-plane orbitals in the moreconducting SLs. Accordingly, when moving through the MIT into the metallic regime a transfer of spectral weight from the locally screened component B to the nonlocally screened component A is observed [Fig. 11(b)].

\section{DISCUSSION}

Structural characterization confirms the formation of sharp IFs observed in previous STEM results [11]. The combination of $\mathrm{Cu}$ and $\mathrm{Mn} L$-edge XAS/XLD and Ca $2 p$ spectra supports

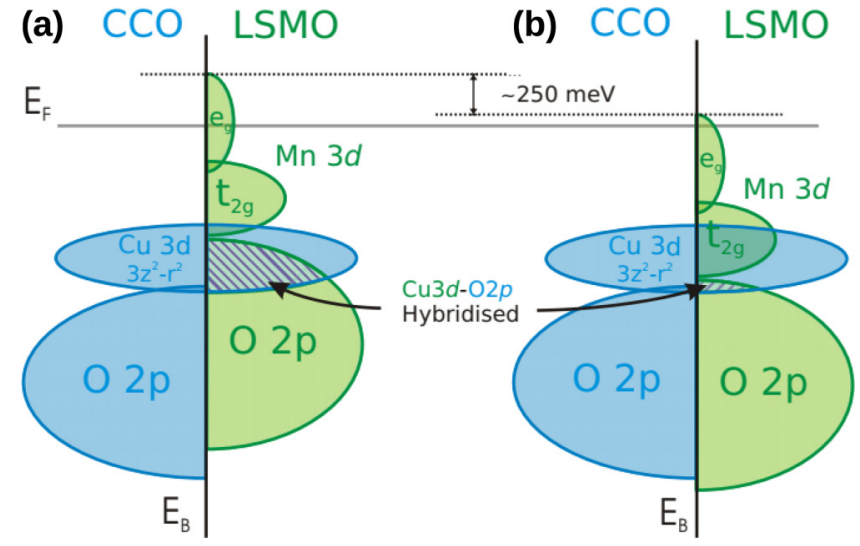

FIG. 13. A schematic diagram of the energy levels at the IF between the CCO (blue) and LSMO (green). (a) Nonmetallic SLs (i.e., thin LSMO blocks or above MIT) and (b) metallic SLs (i.e., thick LSMO blocks or below MIT). Due to the band shift of the LSMO the energy overlap between the $\mathrm{Cu} 3 d$ and $\mathrm{O} 2 p$ orbitals decreases.

the incorporation of oxygen at the IF. The LSMO units in CCO/LSMO SLs exhibit a MIT as a function of sample temperature and LSMO thickness. Transport measurements (Fig. 6) confirm that SLs with thicker LSMO blocks are more metallic at lower temperature. Consistent results were found in VB, Mn $3 s$, and Mn $2 p$ HAXPES spectra (Figs. 7-9). The Mn $3 d e_{g}$ orbitals are increasingly populated as the LSMO thickness rises, resulting in a reduced Mn valence and more metallic behavior. Notably, we observed a downwards band shift in the LSMO bands relative to CCO for SLs below the MIT. This thickness and temperature dependence of the LSMO electronic properties is similar to that of LSMO thin films exhibiting an insulating state driven by the formation of a "dead layer" at the IF to the substrate. Similar results were presented by Adamo et al. for different manganite SLs [21].

Corresponding changes in the spectral features of $\mathrm{CCO}(\mathrm{Cu}$ $2 p$ HAXPES and $\mathrm{Cu} L_{3}$-edge XAS) appear to be driven by the different electronic structures of LSMO above and below the MIT. The absence of temperature dependence of the $\mathrm{Cu}$ $2 p$ HAXPES spectra for the $3 \times 4$ SL [cf. Fig. 11(a)] that does not exhibit MIT testifies that the effect of temperature on the CCO blocks itself is minimal. Since the CCO layer thickness and growth conditions are the same for all SOC SLs, no modifications in the electronic structure would be expected that would explain the observed modifications. Therefore, the electronic structure of the $\mathrm{CCO}$ unit is modified by electronic reconstructions via bridging oxygen at the IF and interaction with the LSMO units.

The evolution of the $\mathrm{Cu} 2 p$ spectra with temperature and LSMO thickness is associated mainly with the variation of the $\mathrm{Cu} 3 d-\mathrm{O} 2 p$ hybridization strength $T$ (cf. Fig. 12). Namely, we observe a decreased hybridization strength for SLs below the MIT, which is also supported by the systematic increase of XLD signal for thicker LSMO blocks as shown in Fig. 4(b). A possible interpretation for the change of the hybridization strength $T$ can be derived from the band alignment between CCO and LSMO blocks (Fig. 10). The energy levels of the $\mathrm{VB}$ at the CCO/LSMO IF are shown schematically in Fig. 13. 
For an insulating SL (e.g., $3 \times 4$ or $3 \times 17$ at $300 \mathrm{~K}$ ), the $\mathrm{Mn}$ $3 d$ bands are partially filled, resulting in an upwards band shift of LSMO. Energy overlap of the interfacial O $2 p$ and $\mathrm{Cu} 3 d$ orbitals allows for strong $\mathrm{Cu} 3 d-\mathrm{O} 2 p$ hybridization (i.e., $T=2.5 \mathrm{eV}$ for $3 \times 4 \mathrm{SL}$ ) as shown in Fig. 13(a). In the conducting SLs (e.g., $3 \times 17$ at $60 \mathrm{~K}$ ), the Mn $3 d$ bands are more populated, resulting in a downwards band shift of LSMO. Consequently, the energy overlap of the interfacial O $2 p$ and $\mathrm{Cu} 3 d$ orbitals decreases, resulting in weaker $\mathrm{Cu} 3 d-\mathrm{O}$ $2 p$ hybridization (i.e., $T=2.15 \mathrm{eV}$ for $3 \times 17 \mathrm{SL}$ at $60 \mathrm{~K}$ ) as shown in Fig. 13(b).

\section{CONCLUSION}

The growth conditions of CCO/LSMO SLs result in distinct IFs: (i) an IF involving a bare Ca layer for MOC SLs and (ii) an IF involving $\mathrm{Ca}$ with planar oxygen coordination for the SOC SLs. The oxygen in the $\mathrm{Ca}$ plane is apical to the $\mathrm{Cu}$ and a bridging oxygen between $\mathrm{Cu}$ and $\mathrm{Mn}$, and is hybridized with the $3 d_{z^{2}-r^{2}}$ orbitals of the transition metals.

The electronic structure in the manganite undergoes a MIT as a function of temperature and LSMO thickness. The transition is characterized by a gradual filling of $\mathrm{Mn} 3 d$-derived states which results in a downwards band shift for the more metallic SLs, and the emergence of the well-screened state in the Mn $2 p$ HAXPES spectra. Furthermore, the Mn valence is decreased towards the value of optimally doped LSMO.
The change in valence state is mainly driven by the thickness of the LSMO and is interpreted in analogy to the dead-layer formation well known for LSMO thin films and SLs.

Systematic changes in the $\mathrm{Cu} L_{3}$-edge XAS/XLD and $\mathrm{Cu} 2 p$ HAXPES core-level spectra and their screening features were observed. The spectra give evidence of a decreased $\mathrm{Cu} 3 d-\mathrm{O} 2 p$ hybridization for conductive SLs below the MIT of the LSMO blocks. The lower hybridization strength is interpreted as a result of a decreased energy overlap of interfacial $\mathrm{O} 2 p$ orbitals with the $\mathrm{Cu} 3 d$ orbitals in the $\mathrm{CCO}$ blocks. The increase of the nonlocal screening channel in the $\mathrm{Cu} 2 p 3 d^{10} \mathrm{~L}$ main peak is indicative of an increased population of more localized in-plane $\mathrm{Cu} 3 d$ orbitals.

Our experiments show that orbital reconstructions at cuprate-manganite IFs can be efficiently tuned via the band alignment of the constituent blocks. The possibility to control the orbital hybridization at cuprate-manganite IFs is opening new routes towards the rational design of oxide heterostructures with engineered functional properties.

\section{ACKNOWLEDGMENTS}

We are particularly grateful to Dave McCue for his outstanding technical assistance at I09. This work was partially supported by the Italian MIUR Grant No. PRIN 20094W2LAY, "Ordine orbitale e di spinnelle eterostrutture di cuprati e manganiti."
[10] D. Samal, H. Tan, H. Molegraaf, B. Kuiper, W. Siemons, S Bals, J. Verbeeck, G. Van Tendeloo, Y. Takamura, E. Arenholz et al., Phys. Rev. Lett. 111, 096102 (2013).

[11] N. Yang, D. Di Castro, C. Aruta, C. Mazzoli, M. Minola, N. B. Brookes, M. Moretti Sala, W. Prellier, O. I Lebedev, A. Tebano et al., J. Appl. Phys. 112, 123901 (2012).

[12] C. Aruta, G. Ghiringhelli, C. Dallera, F. Fracassi, P. G. Medaglia, A. Tebano, N. B. Brookes, L. Braicovich, and G. Balestrino, Phys. Rev. B 78, 205120 (2008).

[13] D. Di Castro, M. Salvato, A. Tebano, D. Innocenti, C. Aruta, W. Prellier, O. I. Lebedev, I. Ottaviani, N. B. Brookes, M. Minola et al., Phys. Rev. B 86, 134524 (2012).

[14] D. Di Castro, C. Aruta, A. Tebano, D. Innocenti, M. Minola, M. M. Sala, W. Prellier, O. Lebedev, and G. Balestrino, Supercond. Sci. Technol. 27, 044016 (2014).

[15] R. von Helmolt, J. Wecker, B. Holzapfel, L. Schultz, and K. Samwer, Phys. Rev. Lett. 71, 2331 (1993).

[16] A. Urushibara, Y. Moritomo, T. Arima, A. Asamitsu, G. Kido, and Y. Tokura, Phys. Rev. B 51, 14103 (1995).

[17] Y. Lu, X. W. Li, G. Q. Gong, G. Xiao, A. Gupta, P. Lecoeur, J. Z. Sun, Y. Y. Wang, and V. P. Dravid, Phys. Rev. B 54, R8357 (1996).

[18] J. Z. Sun, W. J. Gallagher, P. R. Duncombe, L. Krusin-Elbaum, R. A. Altman, A. Gupta, Y. Lu, G. Q. Gong, and G. Xiao, Appl. Phys. Lett. 69, 3266 (1996).

[19] J. Park, E. Vescovo, H. Kim, C. Kwon, R. Ramesh, and T. Venkatesan, Nature (London) 392, 794 (1998). 
[20] A. Tebano, C. Aruta, S. Sanna, P. G. Medaglia, G. Balestrino, A. A. Sidorenko, R. De Renzi, G. Ghiringhelli, L. Braicovich, V. Bisogni et al., Phys. Rev. Lett. 100, 137401 (2008).

[21] C. Adamo, C. A. Perroni, V. Cataudella, G. De Filippis, P. Orgiani, and L. Maritato, Phys. Rev. B 79, 045125 (2009).

[22] T. Pincelli, V. Lollobrigida, F. Borgatti, A. Regoutz, B. Gobaut, C. Schlueter, T.-L. Lee, D. Payne, M. Oura, K. Tamasaku et al., Nat. Commun. 8, 16051 (2017).

[23] C. Adamo, X. Ke, H. Q. Wang, H. L. Xin, T. Heeg, M. E. Hawley, W. Zander, J. Schubert, P. Schiffer, D. A. Muller et al., Appl. Phys. Lett. 95, 112504 (2009).

[24] J. Rubio-Zuazo, L. Onandia, P. Ferrer, and G. R. Castro, Appl. Phys. Lett. 104, 021604 (2014).

[25] C. Schlueter, P. Orgiani, T.-L. Lee, A. Y. Petrov, A. Galdi, B. A. Davidson, J. Zegenhagen, and C. Aruta, Phys. Rev. B 86, 155102 (2012).

[26] L. F. Kourkoutis, J. H. Song, H. Y. Hwang, and D. A. Muller, Proc. Natl. Acad. Sci. USA 107, 11682 (2010).

[27] N. Nakagawa, H. Y. Hwang, and D. A. Muller, Nat. Mater. 5, 204 (2006).

[28] H. Boschker, J. Verbeeck, R. Egoavil, S. Bals, G. van Tendeloo, M. Huijben, E. P. Houwman, G. Koster, D. H. A. Blank, and G. Rijnders, Adv. Funct. Mater. 22, 2235 (2012).

[29] C. Aruta, C. Schlueter, T.-L. Lee, D. Di Castro, D. Innocenti, A. Tebano, J. Zegenhagen, and G. Balestrino, Phys. Rev. B 87, 155145 (2013).

[30] G. H. Fecher, B. Balke, A. Gloskowskii, S. Ouardi, C. Felser, T. Ishikawa, M. Yamamoto, Y. Yamashita, H. Yoshikawa, S. Ueda et al., Appl. Phys. Lett. 92, 193513 (2008).

[31] S. Hüfner, Photoelectron Spectroscopy: Principles and Applications, Advanced Texts in Physics (Springer, Berlin, 2003).

[32] T. Böske, K. Maiti, O. Knauff, K. Ruck, M. S. Golden, G. Krabbes, J. Fink, T. Osafune, N. Motoyama, H. Eisaki et al., Phys. Rev. B 57, 138 (1998).
[33] A. Bocquet and A. Fujimori, J. Electron Spectrosc. Relat. Phenom. 82, 87 (1996).

[34] A. Tebano, G. Balestrino, N. G. Boggio, C. Aruta, B. Davidson, and P. G. Medaglia, Eur. Phys. J. B 51, 337 (2006).

[35] S. Tanuma, C. J. Powell, and D. R. Penn, Surf. Interface Anal. 35, 268 (2003).

[36] Z. Zhong, G. Koster, and P. J. Kelly, Phys. Rev. B 85, 121411(R) (2012).

[37] S. Picozzi, C. Ma, Z. Yang, R. Bertacco, M. Cantoni, A. Cattoni, D. Petti, S. Brivio, and F. Ciccacci, Phys. Rev. B 75, 094418 (2007).

[38] K. Horiba, M. Taguchi, A. Chainani, Y. Takata, E. Ikenaga, D. Miwa, Y. Nishino, K. Tamasaku, M. Awaji, A. Takeuchi et al., Phys. Rev. Lett. 93, 236401 (2004).

[39] C. S. Fadley, D. A. Shirley, A. J. Freeman, P. S. Bagus, and J. V. Mallow, Phys. Rev. Lett. 23, 1397 (1969).

[40] V. R. Galakhov, M. Demeter, S. Bartkowski, M. Neumann, N. A. Ovechkina, E. Z. Kurmaev, N. I. Lobachevskaya, Y. M. Mukowskii, J. Mitchell, and D. L. Ederer, Phys. Rev. B 65, 113102 (2002).

[41] S.-J. Oh, G.-H. Gweon, and J.-G. Park, Phys. Rev. Lett. 68, 2850 (1992).

[42] G.-H. Gweon, J.-G. Park, and S.-J. Oh, Phys. Rev. B 48, 7825 (1993).

[43] S. A. Chambers, T. Droubay, T. C. Kaspar, and M. Gutowski, J. Vac. Sci. Technol. B 22, 2205 (2004).

[44] L. Qiao, T. Droubay, T. Kaspar, P. Sushko, and S. Chambers, Surf. Sci. 605, 1381 (2011).

[45] M. A. van Veenendaal, Phys. Rev. B 74, 085118 (2006).

[46] M. A. van Veenendaal, H. Eskes, and G. A. Sawatzky, Phys. Rev. B 47, 11462 (1993).

[47] K. Okada and A. Kotani, Phys. Rev. B 52, 4794 (1995).

[48] A. Koitzsch, J. Fink, M. S. Golden, K. Karlsson, O. Jepsen, O. Gunnarsson, L. L. Miller, H. Eisaki, S. Uchida, G. Yang et al., Phys. Rev. B 66, 024519 (2002). 